\title{
Erratum: Calculating the free energy difference by applying the Jarzynski equality to a virtual integrable system [Phys. Rev. E 98, 022117 (2018)]
}

Liyun Zhu and Jiao Wang $\odot$

Q (Received 14 July 2020; published 27 July 2020)

DOI: 10.1103/PhysRevE.102.019902

There are typographical errors in Eqs. (14) and (B6). Both equations should be

$$
e^{-\beta \Delta F}=\left(V_{\mathrm{B}} / V_{\mathrm{A}}\right)^{N}\left\langle e^{\beta\left(U_{\mathrm{A}}-U_{\mathrm{B}}\right)}\right\rangle_{\mathrm{A}, \mathbf{r}} .
$$

The numerical results of the free energy difference presented as symbols in Fig. 7 were obtained by using this equation. 